\title{
APLICACIÓN DEL ANÁLISIS DE SOBREVIVENCIA \\ AL ESTUDIO DEL TIEMPO REQUERIDO PARA GRADUARSE \\ EN EDUCACIÓN SUPERIOR: \\ EL CASO DE LA UNIVERSIDAD DE COSTA RICA
}

\section{Survival analysis applied to the time frame required for \\ higher education degree attainment: the case of the \\ University of Costa Rica}

\section{EUGENIA GALLARDO ALLEN*, MAURICIO MOLINA-DELGADO** y REBECA CORDERO CANTILLO ${ }^{* * *}$}

Recibido: 18-06-2015

Revisado: 17-12-2015

Aceptado: 18-02-2016

Resumen. En el siguiente artículo se analiza el tiempo requerido para graduarse como bachiller universitario, así como su relación con predictores socio demográficos y de aptitud académica. Se realizó un seguimiento hasta el año 2010 para la población estudiantil que ingresó

\footnotetext{
* Tiene una maestría en Estadística y actualmente sigue estudios de doctorado en Gobierno y Políticas públicas. Se ha desempañado en el Centro de Evaluación Académica y actualmente es profesora de la Escuela de Estadística y es Directora de Extensión de la Universidad de Costa Rica.

** Es doctor en Psicología por la Universidad Aristotélica de Salónica, Grecia. Es catedrático de la Universidad de Costa Rica, profesor de la Escuela de Filosofía e investigador del Instituto de Investigaciones Psicológicas. Actualmente es director de la Maestría en Ciencias Cognoscitivas.

*** Obtuvo su maestría en Estadística por la Universidad de Costa Rica. Ha sido investigadora del Instituto de Investigaciones Educativas y actualmente se desempeña en la Oficina de Atención Socioeconómica de dicha universidad.
} 
entre los años 2000 y 2003 a la Universidad de Costa Rica. Al realizar comparaciones entre las curvas de sobrevivencia, mediante el estimador Kaplan-Meier, se encontró una probabilidad mayor de graduarse tempranamente en las mujeres y en aquellos(as) estudiantes que cursaron sus estudios fuera de la capital del país. Adicionalmente se construyeron modelos de regresión de Cox, encontrándose asociaciones entre el tiempo de graduación y el tipo de colegio de procedencia, la puntuación de una prueba de aptitud académica y la tenencia de beca socioeconómica. Los resultados apuntan a que altos niveles de aptitud académica están relacionados con menores tiempos de duración de la carrera. Por otra parte, el nivel socioeconómico no parece ser determinante en los tiempos de graduación, lo cual se explica porque probablemente la tenencia de beca mitiga el efecto de dicho factor.

Palabras claves: Graduación, análisis de sobrevivencia, Kaplan-Meier, Regresión de Cox, ayuda financiera.

Abstract. The paper analyzes the time frame required for bachelor degree attainment, as well as its relation with socio-demographic variables and academic aptitude. A followup study of students entering the University of Costa Rica between 2000 and 2003 was conducted up until 2010. The data were analyzed using Kaplan-Meier survival curves. $A$ higher probability of early graduation was found in women and students enrolled in branch campuses. Moreover, Cox regression models were estimated, showing a negative correlation between the time frame required for obtaining the bachelor degree, on one hand, and the score obtained in an academic aptitude test, the type of high school (public/private), and whether the student had received financial aid from the university, on the other. The results show a trend in students with higher academic aptitude of successfully finishing their studies earlier. Furthermore, socioeconomic 
Aplicación del análisis de sobrevivencia al estudio del tiempo requerido para graduarse en educación superior: el caso costarricense

condition does not have a significant effect. The authors claim that this pattern is due to the role of the financial aid system of the university.

Key words: Degree Attainment, survival analysis, Kaplan-Meier, Cox regression, financial aid. 
Aplicación del análisis de sobrevivencia al estudio del tiempo requerido para graduarse en educación superior: el caso costarricense

\section{INTRODUCCIÓN}

Los estudios sobre el logro de títulos universitarios han identificado tres tipos de factores asociados (Astin y Oseguera, 2012): aquellos propios de las características del(a) estudiante, las prácticas y políticas institucionales y las contingencias del contexto. Entre los primeros, se destacan aspectos socioeconómicos como el ingreso, el género, características de la institución de educación secundaria de la que procede el estudiante, variables motivacionales y variables actitudinales como los puntajes obtenidos en pruebas de ingreso. En cuanto a las políticas y prácticas de las instituciones de educación superior, es claro que estas pueden contrarrestar algunas desventajas de los estudiantes a nivel individual. Así, aunque está bien documentada la desventaja con que cuentan aquellos estudiantes que cuentan con ingresos bajos, diversos tipos de programas en las universidades pueden paliar esta problemática (Engle y O'Brien, 2007), incluyendo diversas ayudas para financiar los estudios (Bergeron y Flores, 2015; Carrell y Sacerdote, 2013; Carruthers y Özek, 2016). Finalmente, entre las contingencias se cuentan aspectos como el contexto cultural y geográfico, la naturaleza de la carrera, etc.

Aunque existen diversos estudios sobre el efecto de estos factores en el éxito académico, en particular respecto a la obtención del título universitario (comúnmente bachillerato programado para durar cuatro años), en general las investigaciones adolecen de debilidades metodológicas importantes. Por ejemplo, Astin y Oseguera (2005), mediante una muestra nacional de instituciones superiores en los Estados Unidos, identifican varios factores que afectan las probabilidades de graduación; entre ellos, las puntuaciones en las pruebas de admisión (SAT), las notas de secundaria, y el género. Para ello ajustaron modelos de regresión lineal utilizando como variable dependiente si los estudiantes se habían graduado o no. Esta estrategia de análisis resulta claramente problemática ya que dicha variable dependiente es dicotómica, lo cual viola los supuestos de la regresión lineal, de modo que debió emplearse un modelo logístico u otra herramienta de clasificación (Hosmer, Lemeshow y Sturdivant, 2013). Más allá de que los resultados señalan tendencias en los datos, las ecuaciones encontradas resultan muy sensibles a valores relativamente altos en las variables 
Aplicación del análisis de sobrevivencia al estudio del tiempo requerido para graduarse en educación superior: el caso costarricense

independientes. Adicionalmente los resultados no pueden ser interpretados como estimaciones de probabilidad, como en efecto hacen los autores, ya que un modelo lineal puede producir valores por encima de 1 y por debajo de 0 .

Además, aunque es claro que el fenómeno de las graduaciones está ligado al tiempo, en muchas oportunidades esta variable no es tomada en cuenta. En general lo que se ha hecho es obviar este problema considerando las probabilidades 0 proporciones de graduación para un período de años particular; por ejemplo, para cuatro años y seis años (Astin y Oseguera, 2005; Astin y Oseguera, 2012; Engle y O’Brien, 2007). Sin embargo lo más apropiado sería considerar el fenómeno como dependiente de la variable tiempo.

Más allá de los problemas señalados, lo cierto es que investigaciones como las previamente citadas muestran tendencias de interés para analizar factores asociados a las probabilidades de graduación. En otros casos, por ejemplo en informes institucionales, es común depender de estimaciones claramente inapropiadas, como es el caso del cálculo del tiempo de graduación (v.g., CONARE, 1993; CONARE, 2001).

En el presente estudio se analizará el caso de la Universidad de Costa Rica (UCR) en cuanto a factores asociados al tiempo de graduación. Específicamente se analizará el caso de aquellas carreras que otorgan el título de bachiller. El sistema de titulación de la UCR contempla programas de cuatro años tendientes a la obtención del bachillerato. Adicionalmente, algunas carreras cuentan con títulos de licenciatura, para los cuales el(la) estudiante debe aprobar un año adicional de cursos y presentar un trabajo de tesis. También existen diversos programas de posgrado con título de maestría y doctorado. Para la presente investigación se han considerado únicamente los programas de bachillerato, todos ellos con una duración de cuatro años

En Costa Rica el sistema de educación superior está compuesto por instituciones públicas y privadas. Las instituciones públicas son financiadas por mandato constitucional por el Fondo de Financiamiento para la Educación Superior (FEES), el cual en promedio ha sido de 1.2\% del Producto Interno Bruto para el periodo 2000-2014 (Programa Estado de la Nación, 2015). Actualmente existen cinco universidades públicas en el país, siendo la UCR la más grande de ellas y, de hecho, la más grande del país. Es además la institución de educación superior a la que más estudiantes 
Aplicación del análisis de sobrevivencia al estudio del tiempo requerido para graduarse en educación superior: el caso costarricense

intentan ingresar. Cada año cerca de 50.000 estudiantes compiten por 8.000 cupos (Montero, Rojas-Rojas, Negrín-Hernández, Francis-Salazar, 2015).

El ingreso a la UCR depende parcialmente de que los estudiantes aprueben una prueba de aptitud general denominada Prueba de Actitud Académica (en adelante PAA), cuya puntuación va de 0 a 100 en correspondencia con el porcentaje de respuestas correctas (Jiménez-Alfaro, K. y Morales-Fernández, E., 2010). La admisión en la UCR depende además de otros aspectos que se indican en el artículo III del Reglamento del Proceso de Admisión mediante la Prueba de Aptitud Académica (2003), tales como contar con el diploma de bachiller en educación media, concursar en algún programa de estudio y, en algunas carreras, aprobar pruebas específicas establecidas por algunos programas de estudio. Los puntajes de admisión para acceder a las diferentes carreras, que se imparten en la universidad, varían dependiendo de los cupos disponibles cada año. Aunque algunas carreras suelen ser más apetecidas provocando puntajes de corte más altos, estos patrones varían con el tiempo, dependiendo de cambios de preferencias.

La UCR cuenta con un sistema de atención socioeconómica que consiste en la adjudicación de becas socioeconómicas, que eximen del pago total o parcial de la matrícula. La adjudicación de las becas se realiza mediante un sistema de clasificación automática. Dicho sistema considera variables como el ingreso familiar, los gastos familiares, la tenencia de bienes y la institución secundaria de proveniencia (pública o privada) para clasificar a todos los solicitantes en una categoría de nivel socioeconómico que define si se le exonera del pago de matrícula.

La literatura sugiere que tanto la PAA como el sistema de becas podría afectar las probabilidades de graduación de los estudiantes (Engle y O'Brien, 2007). Astin y Oseguera (2012) reportan un efecto significativo de las puntuaciones del SAT sobre las probabilidades de graduarse en cuatro y seis años.

En cuanto al sistema de becas de la UCR, está claro que corresponde al tipo de políticas institucionales tendientes a reducir el efecto negativo del nivel socioeconómico. Sin embargo, es complicado encontrar estudios empíricos comparables, ya que en general las universidades que cuentan con sistemas de ayuda socioeconómica adjudican las becas entre aquellos solicitantes que realizan una solicitud (Engle y 
O'Brien, 2007) de modo que no cuentan, como en el caso de la Universidad de Costa Rica, con información sobre todos los estudiantes. Adicionalmente, es común que las ayudas no se den en términos de exoneración sino de préstamo, lo que hace que en muchos casos quienes más requieren de ayuda decidan no solicitarla (Bergeron y Flores, 2015).

Finalmente, debe considerarse que un problema importante para estimar el tiempo de duración de las carreras en la UCR es el hecho de que no es posible saber cuáles estudiantes desertan de sus estudios. Esto por cuanto no hay una política de excluir a aquellos por bajo rendimiento o por no matricular cursos por períodos largos. Este hecho hace que las estimaciones del tiempo de duración de los estudios se vean afectadas, siendo estas artificialmente altas al incluir estudiantes que nunca concluirán sus estudios.

\section{PROBLEMAS METODOLÓGICOS}

El presente estudio pretende estimar los tiempos de graduación de sus estudiantes para las distintas carreras de la UCR. Una dificultad para realizar este objetivo, es el hecho de no contarse con un mecanismo para excluir estudiantes que en realidad han abandonado sus carreras, por lo que para estimar el tiempo de graduación es también necesario realizar una estimación de la magnitud de la deserción. Adicionalmente se pretende encontrar variables asociadas a los tiempos de graduación.

Tanto el problema de estimar el tiempo que tardan los estudiantes para graduarse como la magnitud de la deserción presentan dificultades metodológicas interesantes. En el primer caso, una aproximación inicial, a menudo empleada en diversos informes (por ejemplo PEN, 2015) sería calcular la media de los tiempos. Para ello resulta necesario excluir aquellos casos en los que, al momento de la investigación, los(as) estudiantes no hayan aún completado sus carreras. En la práctica esto corresponde al cálculo de una media truncada de la cual son eliminados los valores extremos positivos. Sin embargo, para fines de comparación entre subpoblaciones (por ejemplo entre hombres y mujeres) esta estrategia resulta inviable ya que la información eliminada puede tener un efecto diferente en cada una de ellas. Así por ejemplo, 
supóngase que en un estudio la media de los tiempos de graduación es idéntica para hombres y mujeres pero que para el primer grupo el cálculo se basa únicamente en un $25 \%$ de estudiantes que se han graduado en el momento del estudio, mientras que en el segundo grupo un $50 \%$ se ha graduado. En este caso el cálculo de la media no reflejaría el hecho de que las mujeres se gradúan más rápido que los hombres.

Una forma de solucionar el problema es utilizar la mediana $u$ otros cuantiles para este tipo de comparaciones. De este modo, siempre que se logre seguir la población por un período suficientemente largo, puede realizarse el cálculo de estas medidas y emplearlas para comparar subpoblaciones. Sin embargo este enfoque adolece de otras debilidades.

Aparte de los problemas para el cálculo de parámetros, el estudio de los tiempos de graduación debe enfrentar el hecho de que usualmente se cuenta con estudiantes que provienen de diversas cohortes con diferentes años de ingreso a la universidad. Esto causa que, por ejemplo, el seguimiento de un grupo de estudiantes se dé por diez años pero el de otro solo por cinco. En cuanto a la estimación de la deserción, una posibilidad es simplemente eliminar aquellos casos cuyos tiempos de permanencia excedan un cierto límite definido de forma arbitraria; sin embargo este enfoque desconoce el carácter probabilístico del fenómeno. De este modo, conforme pasan los semestres sin que un estudiante matricule cursos, la probabilidad de que deje sus estudios ciertamente aumenta, pero dado el sistema de la UCR es también posible que posteriormente se reincorpore activamente a sus estudios.

Una forma de enfrentar los problemas señalados es mediante el uso del análisis de supervivencia utilizando la técnica conocida como Kaplan-Meier (KM). Este método consiste en el análisis de eventos en el tiempo donde cada participante es seguido durante un tiempo $T_{i}$ hasta el momento donde un evento de interés ocurra o hasta que el tiempo de observación del evento finalice, en cuyo caso se considera que el tiempo $\mathrm{T}_{\mathrm{i}}$ es desconocido, o como se acostumbra llamar, censurado. Además de permitir una estimación de la mediana y los cuantiles, el análisis de sobrevivencia permite estimar la curva de sobrevivencia, así como comparar las curvas de diversas subpoblaciones.

Para los propósitos del presente artículo se utiliza la técnica de KM considerando como el evento de interés la graduación de bachillerato universitario del o la estudiante. 
Aplicación del análisis de sobrevivencia al estudio del tiempo requerido para graduarse en educación superior: el caso costarricense

Además de ajustar el modelo para toda la universidad se consideraron también modelos por facultad de estudio y sede donde se cursan los estudios, ya que la UCR cuenta con nueve sedes, incluyendo la Sede Central Rodrigo Facio.

Por otra parte, resulta también de interés identificar el modo en que diversos factores se asocian con el tiempo de graduación. Mientras que la técnica de KM tiene limitaciones para realizar este tipo de análisis, la Regresión de Cox permite estudiar los efectos de variables métricas sobre los chances de que un(a) estudiante se gradúe en un tiempo dado.

En este artículo interesa analizar si existe alguna relación entre el tiempo requerido para la graduación y la nota de admisión, el colegio de procedencia y la asignación de beca socioeconómica; considerando estas últimas como variables independientes en el modelo de Cox. Adicionalmente se consideraron diferencias por sexo y sede universitaria, variables que serán también incluidas en los modelos.

La nota de admisión corresponde al promedio simple del puntaje obtenido en una Prueba de Aptitud Académica y el promedio de notas de los últimos dos años de educación secundaria. Este promedio es utilizado para seleccionar los(as) estudiantes que son admitidos a la universidad, y se obtiene en una escala 0-100 que luego se transforma a una escala estándar con puntajes entre 200 y 800 (Jiménez-Alfaro y Morales-Fernández, 2010).

Diversos estudios han mostrado una asociación positiva entre medidas de rendimiento académico y el promedio de admisión (Cubero \& Longhi, 1995; JiménezAlfaro y Morales-Fernández, op. cit.). Con el fin de estudiar la validez predictiva de la prueba de admisión a la universidad, algunas de estas investigaciones utilizan como medidas de rendimiento el llamado logro académico, el cual consiste en el número de créditos aprobados por el (la) estudiante multiplicados por la nota obtenida en cada curso. Según nuestro criterio dicha medida incluye dos dimensiones que conviene diferenciar: el desempeño académico y la velocidad del avance de los(as) estudiantes en sus carreras. Mientras que podríamos esperar que el promedio de admisión fuera predictor de ambas dimensiones, también podría darse el caso de que lo fuera únicamente de una de ellas. 
En este estudio pretendemos analizar si la nota de admisión está asociada o no con el tiempo que tarda un o una estudiante en graduarse, por lo que eventualmente nuestra metodología podría ser de utilidad en estudios de validez predictiva.

Otro de los aspectos que podríamos esperar respecto de los tiempos de graduación es que aquellos(as) estudiantes con nivel socioeconómico más bajo enfrentarían mayores dificultades para terminar sus carreras y probablemente muchos deberían trabajar mientras estudian, de modo que podrían tardar más en graduarse. Aunque este estudio no contó con acceso a otros indicadores socioeconómicos, la universidad sí cuenta con información sobre el colegio de procedencia de todo el estudiantado, variable que es conocida como un excelente indicador de nivel socioeconómico. Esta posible relación entre el colegio de procedencia y el tiempo de graduación podría sin embargo verse moderada por la adjudicación de becas. Puesto que la Universidad de Costa Rica cuenta con un sistema de adjudicación de beca socioeconómica a los(as) estudiantes de bajos recursos, podría ser que dichas becas logren atenuar el efecto negativo de un nivel socioeconómico bajo.

A continuación se explican las técnicas utilizadas para la estimación de la curva de sobrevivencia y para el análisis de otras variables asociadas a la probabilidad de graduarse.

\section{ESTIMACIÓN DE LA CURVA DE SOBREVIVENCIA: EL MODELO DE KAPLAN- MEIER}

El estimador KM fue formulado con el fin de tomar en cuenta la censura en el estudio de los tiempos requeridos para que se presente determinado evento. Típicamente se trataría del fallecimiento de un paciente, lo que explica la terminología que suele emplearse (sobrevivencia, riesgo, etc.), sin embargo la técnica puede aplicarse a cualquier evento que pueda presentarse una sola vez durante el tiempo estudiado (Kaplan y Meier, 1958). Considérese la función $\hat{S} t_{(j)}$ que expresa la probabilidad de sobrevivir al tiempo $t_{(j)}$. Según la definición de probabilidad conjunta, el modelo de KM asume que la probabilidad de sobrevivencia al tiempo $t_{(j)}$, es dada por la multiplicación 
de la probabilidad de sobrevivencia del momento previo $j-1$ por la probabilidad condicional de sobrevivencia al tiempo $j$ dado que ha sobrevivido al tiempo $j-1$.

$$
\hat{S} t_{(j)}=\hat{S}\left(t_{j-1}\right) * \hat{P}\left(T>t_{(j)} \mid T>t_{(j-1)}\right)
$$

Sustituyendo $\hat{S}\left(t_{j-1}\right)$ sucesivamente como producto de la función evaluada en tiempos anteriores tenemos que en general la función de sobrevivencia para el tiempo es igual a:

$$
\hat{S} t_{(j)}=\prod_{i=1}^{j} \hat{P}\left(T>t_{(i)} \mid T>t_{(i-1)}\right)
$$

Para la estimación de la función de supervivencia en el tiempo j, Kaplan \& Meier en 1958 propone el siguiente cálculo:

$$
\hat{S} t_{(j)}=\prod_{t i<t} \frac{n_{i}-d_{i}}{n_{i}}
$$

Donde $n_{i}$ representa el número de sujetos en riesgo de falla (en nuestro caso de graduarse) en el tiempo $t_{j}$ y $d_{i}$ el total de los mismos que efectivamente experimentan el evento (se gradúan) en $t_{j}$.

Un aspecto a considerar en el estimador de $\mathrm{KM}$ es el de los datos censurados; es decir, aquellas situaciones en las que no es posible conocer el tiempo en que se presenta el evento. Un caso típico es la denominada censura por la derecha o tipo I, el cual se presenta cuando un individuo no experimenta el evento durante el transcurso del estudio. La censura por la derecha tipo I generalizada se da cuando las unidades entran en el estudio en momentos diferentes pero se ha establecido una fecha en la cual se cerrará el estudio (Gómez, 2004). Así, por ejemplo, en este estudio se analizaron todos los casos de estudiantes que ingresaron a la universidad entre los años 2000 y 2003 donde se observó el evento de graduación de bachillerato hasta el año 2010. Para la fecha de cierre del estudio, los(as) estudiantes que ingresaron en el 2000 tenían diez años en la universidad por lo que previsiblemente muchos de ellos(as) 
podían haber concluido sus estudios; en cambio los que ingresaron en el 2003 tenían solo siete años, de manera que en muchos de esos casos podría no observarse el evento, teniendo que ser tratado como censurado.

El estimador de KM trata los casos de censura eliminándolos de la base para estimación de la probabilidad en el momento en que no se tenga información sobre si se ha dado el evento o no. Para el ejemplo anterior, los(as) estudiantes que ingresaron en el 2003 formarían parte de la $n_{i}$ que aparece en la ecuación (3), en los tiempos correspondientes a los siete años en que fueron observados, pero a partir del semestre siguiente serían eliminados restándose los casos donde el evento graduación no se ha dado de la magnitud de $n_{\text {i }}$.

Para realizar la comparación entre las curvas de sobrevivencia de dos o más subpoblaciones se utiliza la prueba log-rank. Para el caso de dos curvas se parte de la hipótesis nula de que las curvas son iguales. El estadístico utilizado es una Chicuadrada con $i-1$ grados de libertad:

$$
\log _{-} \operatorname{rank}=\frac{\left(\sum_{j=1}^{n}(m i j-e i j)\right)^{2}}{\operatorname{Var}\left(\sum_{j=1}^{n}(m i j-e i j)\right)}
$$

donde $m_{i j}$ representa el número de fallos del grupo $i$ en el tiempo $j$ y $e_{i j}$ el número de fallos esperados del grupo $i$ en el tiempo $j$.

En el número de fallos esperados se calcula la proporción en riesgo por el número de eventos que ocurren en ambos grupos. Por ejemplo para el caso de dos grupos el valor esperado para el grupo 1 en el tiempo $j$ se calcula de la siguiente forma:

$$
e_{1 j=\left(\frac{n_{1 j}}{n_{1 j}+n_{2 j}}\right) *\left(m_{1 j+m_{2 j}}\right)}
$$

\section{REGRESIÓN DE COX}

La Regresión de Cox es una técnica utilizada en análisis de sobrevivencia con el fin de considerar el efecto de un grupo de variables independientes $\left(x_{1}, x_{2}, x_{3}, \ldots . x_{p}\right)$ sobre las Revista Páginas de Educación. Vol. 9, Núm. 1 (2016) ISSN: 1688-5287; e-ISSN: 1688-7468 
probabilidades de ocurrencia de un determinado evento. El modelo asume la presencia de dos componentes independientes que determinan el riesgo de ocurrencia del evento: uno que es función del tiempo denominado $h_{0}(t)$ y el otro que no depende del tiempo sino que es una función exponencial de las variables predictoras (Cox, 1972). Bajo el supuesto de que ambos componentes son independientes, se considera que el riesgo viene dado por la multiplicación de los mismos, obteniéndose la Ecuación (6).

$$
h(t, X)=h_{0}(t) e^{\Sigma_{i=1}^{n} \beta_{i} x_{i}}
$$

En realidad el componente $h_{0}(t)$ no se utiliza en el análisis, siendo los valores de los coeficientes $\beta_{i}$ los que interesan. Desarrollando la ecuación (6) podemos obtener la expresión en (7).

$$
h(t, x)=h_{0} e^{\beta_{1} x_{1}} e^{\beta_{2} x_{2}}
$$

Luego, para el caso donde $x_{1}$ es una variable dicotómica, si calculamos la razón de momios para $x_{1}=1$ respecto de $x_{1}=0$, esta viene dada por la expresión en (8)

$$
\frac{h(t, x=1)}{h(t, x=0)}=\frac{e^{\beta_{1}}}{e^{0}}=e^{\beta_{1}}
$$

Nótese que (8) no depende de $t$. Dicho resultado puede ser generalizado para el caso de variables métricas, en cuyo caso la razón de momios se interpreta como el factor en que se multiplica el riesgo por cada unidad de cambio en $x_{1}$.

La significancia estadística de los coeficientes puede ser evaluada mediante el estadístico de Wald, la cual considera la hipótesis nula de que los coeficientes planteados en el modelo sean diferentes de cero, es decir, de que el exponencial del coeficiente sea diferente de 1 . El estadístico de Wald se obtiene dividiendo el coeficiente estimado $\left(\beta_{i}\right)$ entre el error estándar de la estimación.

\section{ESTRATEGIA DE ANÁLISIS}


Para el presente estudio se analizó toda la población estudiantil que ingresó a la Universidad de Costa Rica entre los años 2000 y 2003. Considerando como evento de interés la graduación de bachillerato universitario se realizó un seguimiento hasta el año 2010. Para ello se utilizaron las bases de la Oficina de Registro e Información (ORI) y las bases de asignación de becas socioeconómicas de la Oficina de Becas y Atención Socioeconómica (OBAS) de dicha universidad, para esos mismos años. El tiempo asociado al evento de interés fue considerado en términos del número de semestres. Se aplicó la técnica de análisis de Sobrevivencia de Kaplan-Meier para analizar el patrón de duración de las carreras de los estudiantes.

Además de estudiar las curvas de sobrevivencia para la totalidad de los estudiantes de la universidad, se realizaron análisis independientes por cada una de las facultades, entre la Sede Rodrigo Facio y las sedes o recintos regionales, así como por sexo. Sólo se tomaron en cuenta las carreras que otorgan el título de grado de Bachiller. El plan de estudio de todas las carreras de grado de Bachillerato tiene una duración de ocho semestres por lo que en principio pueden ser comparados los tiempos reales de graduación de los(as) estudiantes.

Adicionalmente se analizaron posibles diferencias en los tiempos de graduación considerando los promedios de admisión, la asignación de beca socioeconómica y el sexo de los(as) estudiantes. Para ello se trabajó con un modelo de Regresión de Cox.

Los análisis fueron realizados en el programa $R$ versión 2.13.0, mediante el uso de las bibliotecas survivaly splines.

\section{RESULTADOS}

\section{Descripción de la población estudiada}

Un $49.47 \%$ de la población estudiada está constituida por mujeres. El $65.61 \%$ de dicha población provenía de colegio público y a un $57.69 \%$ de la misma se le había otorgado algún tipo de ayuda mediante una beca socioeconómica 
Estos porcentajes se mantuvieron aproximadamente iguales independientemente de la cohorte según año de ingreso de los(as) estudiantes. La media del promedio de admisión fue de $579.70(\mathrm{DE}=69.25)$ y tampoco se encontraron diferencias significativas por año de ingreso.

\section{Análisis descriptivo de la censura presente en las variables}

Los Cuadros 1, 2, 3 y 4 muestran información sobre el número de estudiantes, eventos (graduaciones) y censuras observadas respectivamente por sexo, sede (o recinto) de la universidad y facultad.

Al analizar la variable sexo se tiene que al momento de finalizar el periodo de observación no se había graduado el $53 \%$ de las mujeres ni el $67 \%$ de los hombres (Cuadro 1).

Cuadro 1. Total de censuras y eventos observados (graduación de bachillerato) por sexo. Universidad de Costa Rica.

\begin{tabular}{lcccc}
\hline \multirow{2}{*}{ Sexo } & & Número de & \multicolumn{2}{c}{ Censuras } \\
\cline { 4 - 5 } & $\mathrm{N}$ & Eventos & Absoluto & Porcentaje \\
\hline Mujer & 7353 & 3491 & 3862 & 52,52 \\
Hombre & 7511 & 2507 & 5004 & 66,62 \\
\hline Total & 14864 & 5998 & 8866 & 59,65 \\
\hline
\end{tabular}

Fuente: Elaboración propia.

Respecto a las sedes o recintos, el Cuadro 2 muestra que, en tres de ellas, se graduó más del $50 \%$ durante el período. Es el caso de la Sede de San Ramón (51\%), el Recinto de Tacares (54\%) y la Sede del Atlántico (59\%). Para las demás sedes o recintos en el periodo de observación no se llegó a graduar el $50 \%$ de la población estudiantil.

Cuadro 2. Total de censuras y eventos observados (graduación de bachillerato) por sede o recinto. Universidad de Costa Rica.

\begin{tabular}{lll}
\hline Sede o Recinto $\quad$ Número de $\quad$ Censuras \\
\end{tabular}


Aplicación del análisis de sobrevivencia al estudio del tiempo requerido para graduarse en educación superior: el caso costarricense

\begin{tabular}{lrrrr}
\hline & $N$ & Eventos & Absoluto & Porcentaje \\
\cline { 3 - 4 } & & & & \\
\hline Rodrigo Facio & 11296 & 4280 & 7016 & 62,11 \\
San Ramón & 1150 & 583 & 567 & 49,30 \\
Tacares & 326 & 177 & 149 & 45,71 \\
Atlántico & 348 & 205 & 143 & 41,09 \\
Paraíso & 200 & 98 & 102 & 51,00 \\
Guápiles & 188 & 74 & 114 & 60,64 \\
Liberia & 776 & 348 & 428 & 55,15 \\
Limón & 188 & 79 & 109 & 57,98 \\
Pacífico & 392 & 154 & 238 & 60,71 \\
\hline Total & 14864 & 5998 & 8866 & 59,65 \\
\hline
\end{tabular}

Fuente: Elaboración propia.

En cuanto a las censuras por facultad a la que pertenece el o la estudiante al momento de finalizar la observación, sólo en la Facultad de Medicina se graduó más del $50 \%$ de los y las estudiantes que ingresaron en los años 2000, 2001, 2002, 2003 (Cuadro 3).

Cuadro 3. Total de censuras y eventos observados (graduación de bachillerato) por facultad. Universidad de Costa Rica

\begin{tabular}{lrrrr}
\hline \multirow{2}{*}{ Facultad } & & Número & \multicolumn{2}{c}{ Censuras } \\
\cline { 5 - 6 } & & Eventos & Absoluto & Relativo \\
\hline Bellas Artes & 346 & 168 & 178 & 51,45 \\
Letras & 1115 & 234 & 881 & 79,01 \\
Ciencias & 998 & 221 & 777 & 77,86 \\
Derecho & 825 & 296 & 529 & 64,12 \\
Educación & 1792 & 733 & 1059 & 59,10 \\
Ciencias Económicas & 3159 & 1361 & 1798 & 56,92 \\
Ciencias Sociales & 1745 & 781 & 964 & 55,24 \\
Ingeniería & 1655 & 616 & 1039 & 62,78 \\
Medicina & 686 & 435 & 251 & 36,59 \\
Sedes Regionales & 1988 & 978 & 1010 & 50,80 \\
Ciencias & 555 & 175 & 380 & 68,47 \\
Agroalimentarias & & & &
\end{tabular}

Revista Páginas de Educación. Vol. 9, Núm. 1 (2016) ISSN: 1688-5287; e-ISSN: 1688-7468 
Aplicación del análisis de sobrevivencia al estudio del tiempo requerido para graduarse en educación superior: el caso costarricense

\begin{tabular}{|c|c|c|c|c|}
\hline Total & 14864 & 5998 & 8866 & 59,65 \\
\hline
\end{tabular}

Finalmente, en el grupo de los(as) estudiantes que no poseen beca socioeconómica el porcentaje de censura resultó de $66.19 \%$, lo que quiere decir que al momento de finalizar el periodo de observación solo se había graduado el $34 \%$ frente a un $45 \%$ en el grupo de los que sí disfrutaron de alguna beca socioeconómica por parte de la universidad (Cuadro 4).

Cuadro 4. Total de censuras y eventos observados (graduación de bachillerato) por tenencia de beca socioeconómica. Universidad de Costa Rica.

\begin{tabular}{|c|c|c|c|c|}
\hline \multirow{2}{*}{$\begin{array}{l}\text { Beca } \\
\text { Socioeconómica }\end{array}$} & \multirow{2}{*}{ Total N } & \multirow{2}{*}{$\begin{array}{l}\text { Número } \\
\text { Eventos }\end{array}$} & \multicolumn{2}{|c|}{ Censura } \\
\hline & & & $\mathrm{N}$ & Porcentaje \\
\hline No tiene & 6289 & 2126 & 4163 & 66,19 \\
\hline Sí tiene & 8575 & 3872 & 4703 & 54,85 \\
\hline Total & 14864 & 5998 & 8866 & 59,65 \\
\hline
\end{tabular}

Fuente: Elaboración propia.

\section{Análisis y comparación de la curvas de sobrevivencia}

Al analizar la curva de sobrevivencia para el tiempo de graduación (Figura 1) se encontró que la probabilidad de que un(a) estudiante no se gradúe en 16 semestres es de .63, lo que quiere decir que en dicho período (16 semestres) se espera que únicamente se gradúe el $37 \%$ de la población. 


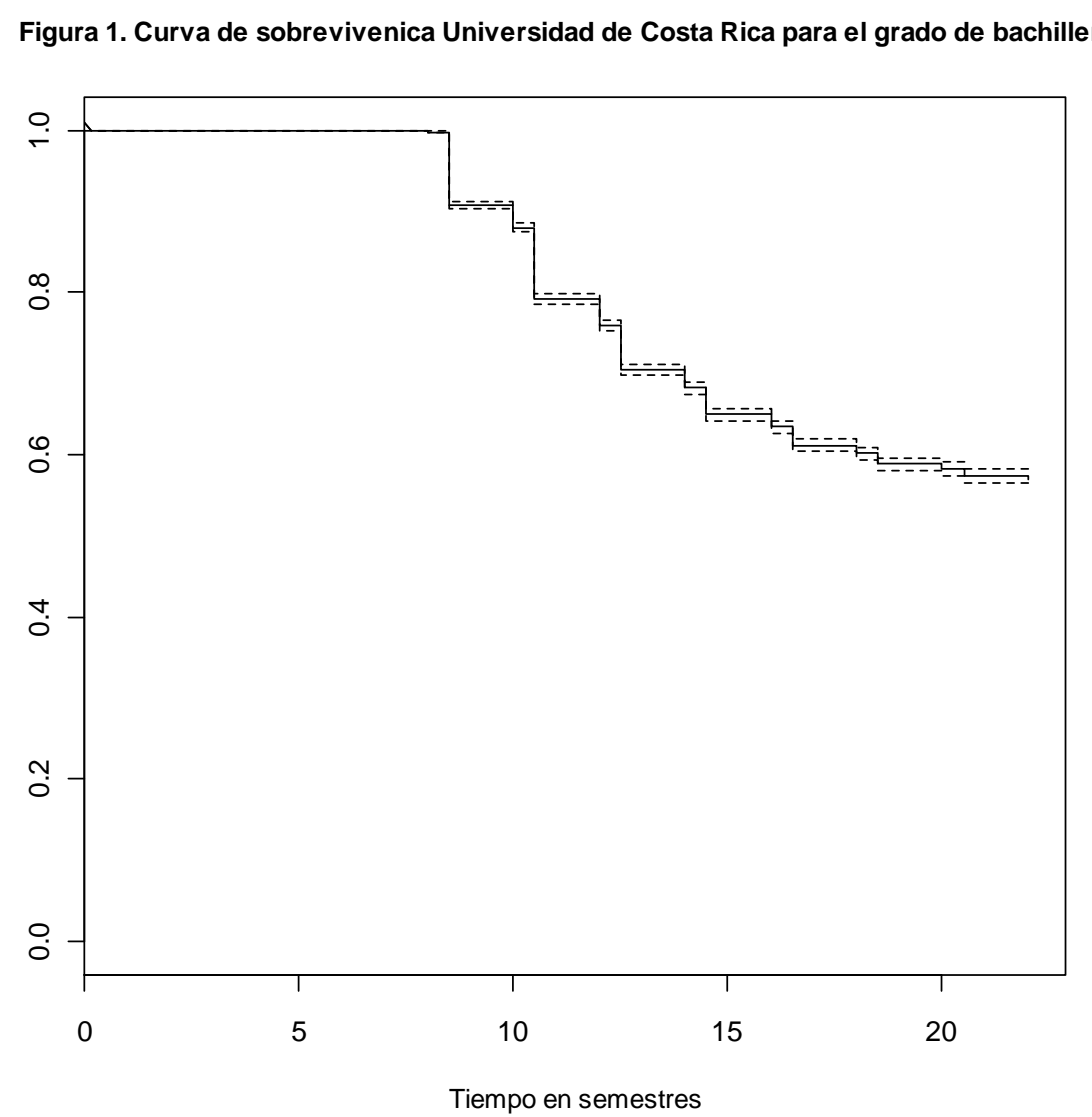

Un problema con estas estimaciones es que, al no contar la Universidad de Costa Rica con un sistema para reconocer cuáles estudiantes han desertado de sus estudios, los porcentajes de graduación se ven afectados al incluir en su base a dichos estudiantes. Para paliar este problema, se utilizaron los resultados del análisis de sobrevivencia con el fin de estimar el porcentaje de deserción. Esto representa en sí mismo un resultado de interés, ya que la universidad no cuenta con estadísticas precisas al respecto. Para ello asumimos que la curva de sobrevivencia sigue un patrón que se ajusta a una distribución exponencial pero que en lugar de tener asintóticamente a 0 (es decir que para valores altos de la variable tiempo el número de estudiantes no graduados se acerca a cero) tiende a un valor que representa el porcentaje de estudiantes que no concluirán sus estudios, es decir, aquellos que desertaron de sus estudios. De este modo, al ajustar los datos a un modelo como el descrito anteriomente, 
se obtiene una ecuación como la mostrada en (9), donde $S$ representa el porcentaje de estudiantes que no se han graduado antes del tiempo $t$.

$$
S(t)=B 0+e-b 1 t
$$

Al calcular el límite de $S(t)$ cuando $t$ tiende a infinito, se obtiene la estimación del porcentaje de deserción, correspondiente a BO. Mediante el uso del paquete $n / r e g$ de $R$, se obtuvo la siguiente ecuación:

$$
S(x)=.38074-\exp (-.08967 t)
$$

De este modo, la estimación del porcentaje de deserción de los estudios universitarios sería del $38.07 \%$. Ajustando los resultados asumiendo dicho porcentaje de deserción, el 37\% que según la estimación inicial se graduaría en 16 semestres se convierte en un $65 \%$ respecto de la base de estudiantes que no dejarían sus estudios. Del mismo modo, aunque al realizar el análisis con la totalidad de la muestra no es posible calcular la mediana, ya que para el período analizado solo se alcazó el $47 \%$ de las graduaciones, al ajustar las estimaciones considerando la deserción, el 50\% de los estudiantes se graduarían entre 16 y 17 semestres, es decir al octavo año de estudios.

Por otra parte, al considerar el sexo de los(as) estudiantes, la prueba de log-rank mostró que el tiempo que tardan en graduarse las mujeres es significativamente inferior al de los hombres, $X^{2}(1)=364,20, p<.01$, tal y como se muestra en la Figura 2. 
Figura 2. Curva acumulada de la probabilidad de graduarse por sexo

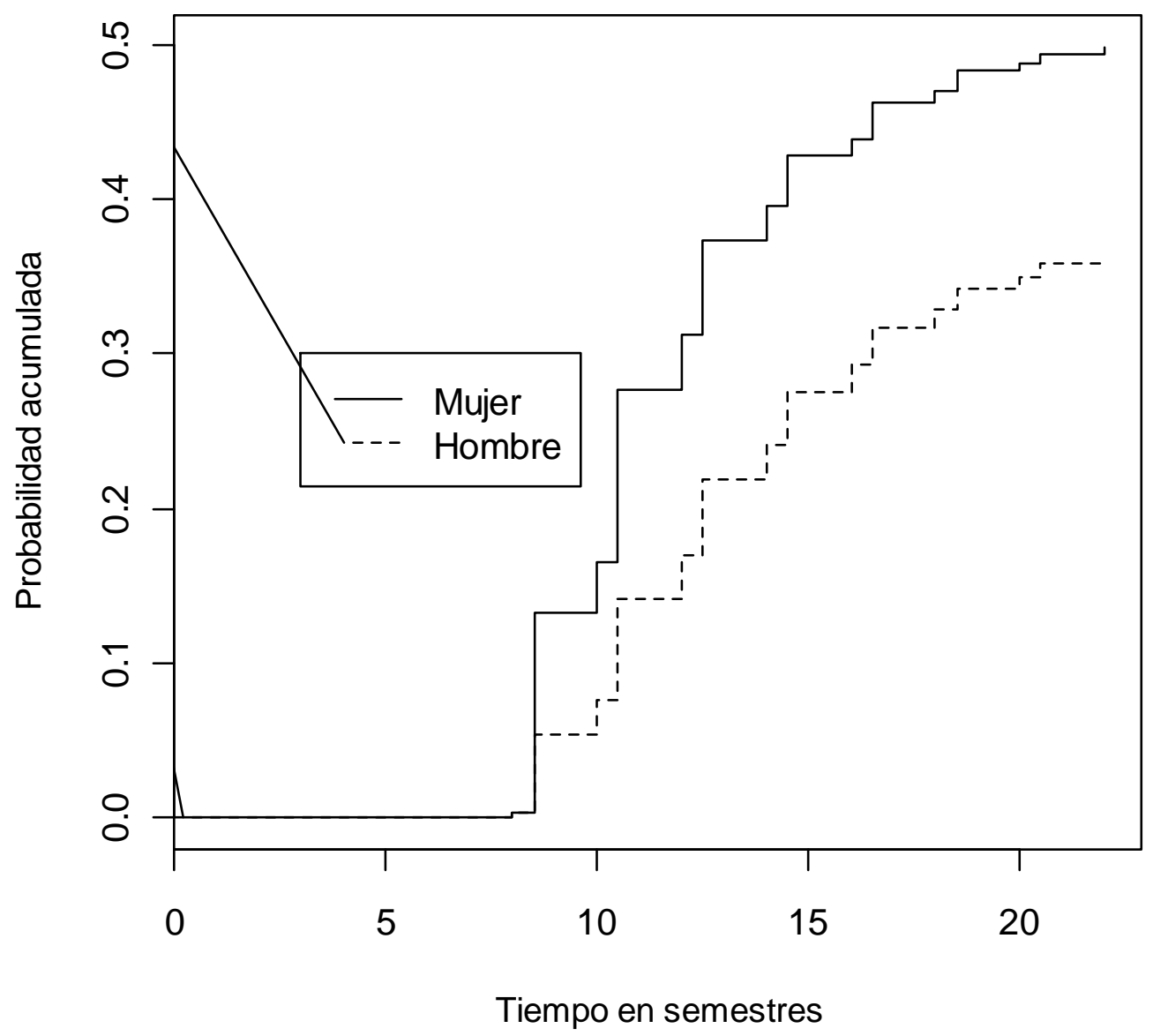

Un análisis comparativo por sede o recintos donde cursa la carrera el o la estudiante utilizando nuevamente la prueba de log-rank, dio evidencia de diferencias significativas entre la Sede central Rodrigo Facio, ubicada en la capital del país, y el resto de las sedes o recintos alejados de dicha ciudad, $X^{2}(1)=186,889, p<.01$.

Al compararse las curvas de sobrevivencia por Facultad se encontraron diferencias estadísticamente significativas, $X^{2}(10)=638,402, p<0,01$. La Facultad que tiene la probabilidad más elevada de graduarse es la de Medicina con .55 para 16 ciclos lectivos o menos, mientras que la menor es Ciencias Básicas con una probabilidad de .19 (ver Figura 3). 
Aplicación del análisis de sobrevivencia al estudio del tiempo requerido para graduarse en educación superior: el caso costarricense

Figura 3. Curva acumulada de la probabilidad de graduarse por Facultad

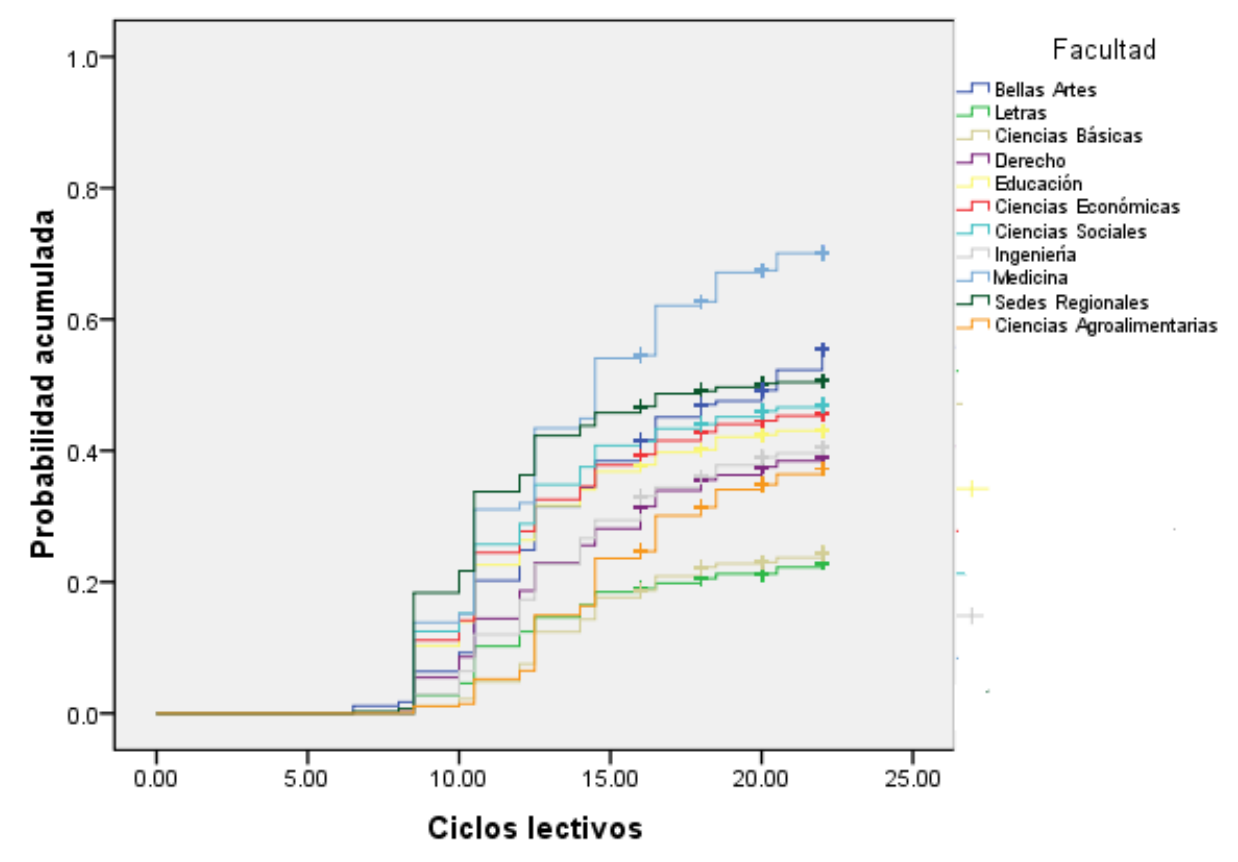

Finalmente, al comparar las curvas correspondientes a aquellos que disfrutaron de beca socioeconómica con respecto a los que no lo hicieron, nuevamente la diferencia entre las curvas resultó significativa en la prueba de log-rank. Como ejemplo, si un(a) estudiante no contó con beca su probabilidad de graduarse en 15 ciclos lectivos o menos fue de .3 contra una probabilidad de .42 en el caso de que no contara con dicha ayuda (ver Figura 4). 
Aplicación del análisis de sobrevivencia al estudio del tiempo requerido para graduarse en educación superior: el caso costarricense

Figura 4. Curva acumulada de la probabilidad de graduarse con y sin beca

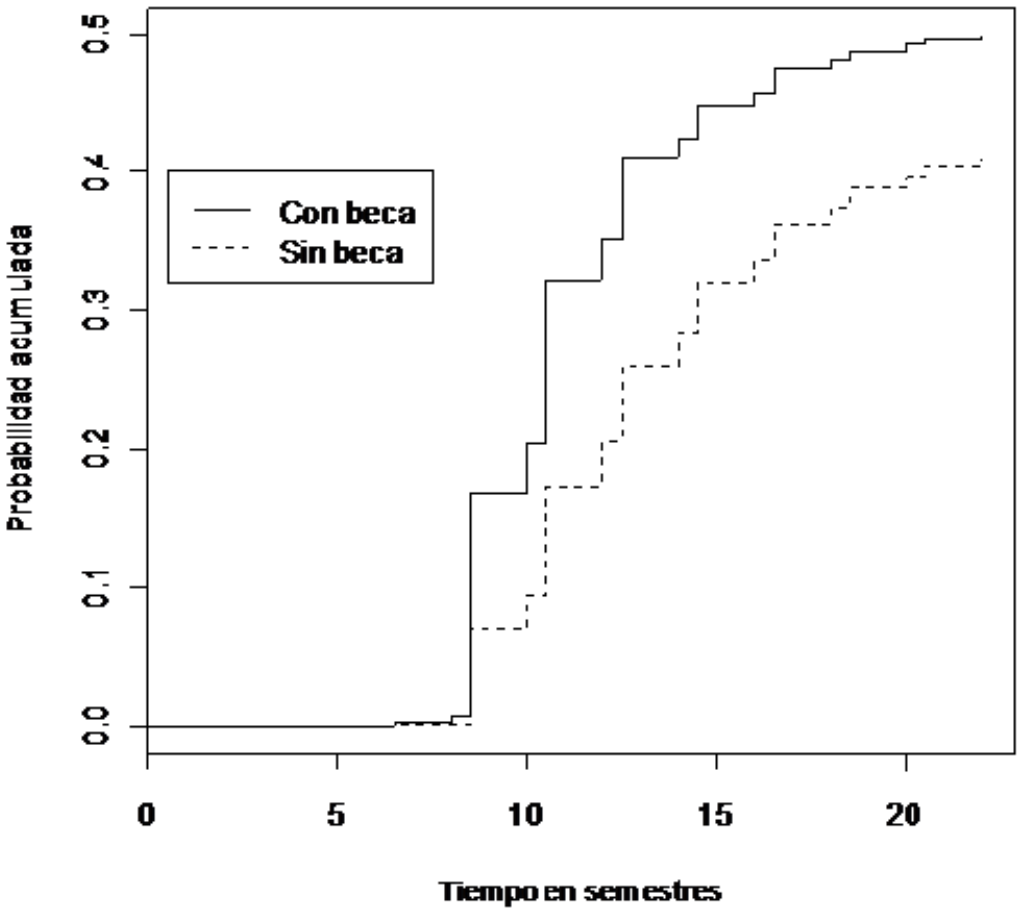

\section{Factores asociados al tiempo de graduación}

Para estudiar factores asociados al tiempo de graduación se empleó una serie de modelos de regresión de Cox. El primer modelo se muestra en la ecuación (11) y consideró el efecto de tres variables: el colegio de proveniencia del(a) estudiante (público o no público), su nota de admisión (la cual consiste en el promedio de la nota de una prueba de habilidades generales y las notas de los dos últimos años de secundaria), y finalmente el sexo del(a) estudiante.

$$
H(t)=H(t 0) \cdot e^{\left(B_{1} \cdot \text { Colegio }+B_{2} \cdot \text { Admisión }+B_{3} \cdot \text { Sexo }\right)}
$$

Como se muestra en el Cuadro 5, los efectos correspondientes a las tres variables resultaron significativos en la prueba de Wald. El exponencial de los coeficientes mostró que los(as) estudiantes provenientes de colegio público (y por tanto gratuito) tenían un $30 \%$ más de riesgo de graduarse respecto de aquellos(as) de Revista Páginas de Educación. Vol. 9, Núm. 1 (2016) ISSN: 1688-5287; e-ISSN: 1688-7468 
Aplicación del análisis de sobrevivencia al estudio del tiempo requerido para graduarse en educación superior: el caso costarricense

colegios no públicos (de pago). Este resultado es sorprendente ya que podemos considerar al tipo de colegio de proveniencia como un indicador del nivel socioeconómico de los(as) estudiantes de modo que, contrario a lo encontrado, sería de esperar que quienes provienen de colegios privados tengan un mayor nivel socioeconómico y se gradúen más rápido.

Los resultados respecto de la nota de admisión en cambio fueron coherentes con lo esperado. Por cada aumento de 100 puntos en dicha nota (cuyo valor máximo es de 800 ) se encontró que el riesgo de graduarse aumenta en un $42 \%$.

Finalmente, el ser hombre implica que el riesgo de graduarse es de 0,58 con respecto de ser mujer, es decir, $42 \%$ menor que el de las mujeres.

Cuadro 5. Coeficientes de la regresión de Cox para el modelo 1

\begin{tabular}{lcccccc}
\hline \multirow{2}{*}{ Variables } & \multirow{2}{*}{$\mathrm{B}$} & $\exp (\mathrm{B})$ & $\operatorname{se}(\mathrm{B})$ & $\mathrm{z}$ & \multicolumn{2}{c}{ Intervalo de Confianza } \\
& & & & & Inferior & Superior \\
\hline Colegio & 0,26 & $1,296^{* * *}$ & 0,0282 & 9,2 & 1,2267 & 1,3701 \\
Admisión & 0,348 & $1,416^{* * *}$ & 0,0188 & 18,5 & 1,3648 & 1,4692 \\
Sexo & $-0,548$ & $0,578^{* * *}$ & 0,0263 & $-20,8$ & 0,5489 & 0,6086 \\
\hline
\end{tabular}

Fuente: Elaboración propia.

Un segundo modelo fue empleado para entender mejor el patrón encontrado. En este se incluyó, además de las variables del primer modelo si el(la) estudiante había disfrutado de una beca de asistencia socioeconómica (ecuación 12). Dicha beca corresponde a una exhoneración (total o parcial) del pago de la matrícula que brinda la Universidad de Costa Rica a aquellos estudiantes que por razones socioeconómicas lo requieren. Con esto se pretendía comprender el hecho contraintuitivo de que los estudiantes provenientes de colegios publicos se graduan más rápido.

$$
H(t)=H(t 0) \cdot e^{\left(B_{1} \cdot \text { Colegio }+B_{2} \cdot \text { Beca }+B_{3} \cdot \text { Admisión }+B_{4} \cdot \text { Sexo }\right)}
$$

Al incorporar la variable de si el(la) estudiante posee beca socioeconómica, el colegio de procedencia no fue estadísticamete significativo, mientras que las demás variables sí lo fueron (Cuadro 6). Los y las estudiantes con beca socioeconómica 
muestran en este modelo un riesgo $63 \%$ mayor de graduarse con respecto a los que no poseen beca. De forma similar respecto del modelo 1, por cada 100 puntos en la nota de admisión hay un riesgo $45 \%$ mayor de graduarse y el ser hombre representa un riesgo de graduarse de 0,58 , es decir $42 \%$ menor que el de las mujeres (Cuadro 6 ).

Estos resultados sugieren que el contar con beca socieconómica es el factor que hace que estudiantes con menor nivel socioeconómico tengan mayores probabilidades de graduarse. Esto puede deberse al hecho de que la beca socioeconómica funcione como una ayuda para que los estudiantes puedan realizar sus estudios satisfactoriamente, o bien puede ser que esta se convierta en una presión para ganar los cursos, dado que en caso contrario la universidad puede disminuir o quitarles la ayuda.

\section{Cuadro 6. Coeficientes de la regresión de Cox para el modelo 2}

\begin{tabular}{lccccc}
\hline \multirow{2}{*}{ Variables } & B & exp(B) & se(B) & $\begin{array}{c}\text { Intervalos de Confianza } \\
\text { Inferior }\end{array}$ & Superior \\
\hline Colegio & $-0,018$ & 0,98168 & 0,034 & 0,92 & 1,05 \\
Beca & 0,491 & $1,63313^{* * *}$ & 0,033 & 1,53 & 1,74 \\
Admisión & 0,374 & $1,45291^{* * *}$ & 0,019 & 1,40 & 1,51 \\
Sexo & $-0,546$ & $0,57905^{* * *}$ & 0,026 & 0,55 & 0,61 \\
\hline$* * *=p<0.001$ & & & & &
\end{tabular}

Fuente: Elaboración propia.

Posteriormente el modelo 2 fue aplicado por separado a los(as) estudiantes de la Sede Rodrigo Facio de la Universidad de Costa Rica y a aquellos pertenecientes a otras sedes, las cuales se encuentran en otros lugares fuera de la capital del país. Para la Sede Rodrigo Facio, lo mismo que para toda la población de estudiantes, el sexo, la nota de admisión y la beca resultaron significativos al 1\%, y los coeficientes muestran efectos similares sobre el riesgo de graduarse a los encontrados en los análisis que incluyen a toda la población, tal y como se muestra en el Cuadro 7. El colegio de procedencia, en cambio, resultó significativo al 5\%. Este último patrón es sin embargo coherente con la explicación de que la beca de asistencia socioeconómica es la causante del resultado inesperado respecto del colegio de procedencia, puesto que al incluirse ambas variables el coeficiente del colegio muestra que los(as) estudiantes de Revista Páginas de Educación. Vol. 9, Núm. 1 (2016) ISSN: 1688-5287; e-ISSN: 1688-7468 
Aplicación del análisis de sobrevivencia al estudio del tiempo requerido para graduarse en educación superior: el caso costarricense

instituciones públicas tienen menores probabilidades de graduarse (un 10\% menos) que aquellos(as) de instituciones no públicas.

Para las sedes regionales en cambio, el colegio de procedencia no resultó estadísticamente significativo, mientras que las otras variables lo fueron al 1\% (Cuadro 7). A partir de estos resultados se puede decir que la variable colegio, en las sedes regionales, no parece aportar valor explicativo al modelo, la variable posesión de beca socioecnómica parece tener una mayor influencia (un aumento del $62 \%$ en el riesgo) en el modelo establecido para las sedes regionales con respecto al modelo establecido para la sede Rodrigo Facio, mientras que la nota de admisión tiene una mayor influencia para el modelo establecido para la Sede Rodrigo Facio con respecto al establecido para las sedes regionales, donde el riesgo aumenta solo el $41 \%$ por cada 100 puntos en la nota de admisión.

Cuadro 7 . Coeficientes de la regresión de Cox por sedes o recintos

\begin{tabular}{|c|c|c|c|c|c|}
\hline \multirow{2}{*}{ Variables } & \multirow{2}{*}{ B } & \multirow{2}{*}{$\exp (B)$} & \multirow{2}{*}{$\operatorname{se}(B)$} & \multicolumn{2}{|c|}{ Intervalos de Confianza } \\
\hline & & & & Inferior & Superior \\
\hline \multicolumn{6}{|c|}{ Sede Rodrigo Facio } \\
\hline Colegio & $-0,106$ & 0,89954 ** & 0,03818 & 0,835 & 0,969 \\
\hline Beca & 0,435 & $1,54435^{* * *}$ & 0,03782 & 1,434 & 1,663 \\
\hline Admisión & 0,509 & $1,66355^{* * *}$ & 0,02247 & 1,592 & 1,739 \\
\hline Sexo & $-0,552$ & $0,57573^{* * *}$ & 0,03113 & 0,542 & 0,612 \\
\hline \multicolumn{6}{|c|}{ Sedes o Recintos Regionales } \\
\hline Colegio & 0,046 & 1,047 & 0,08525 & 0,886 & 1,2376 \\
\hline Beca & 0,480 & $1,61591^{* * *}$ & 0,0727 & 1,401 & 1,8634 \\
\hline Admisión & 0,343 & $1,40863^{* * *}$ & 0,04148 & 1,299 & 1,5279 \\
\hline Sexo & $-0,555$ & $0,57395 * * *$ & 0,04943 & 0,521 & 0,6323 \\
\hline
\end{tabular}

Fuente: Elaboración propia.

Finalmente, el modelo 2 también fue aplicado por separado a las diferentes facultades de la Sede Central Rodrigo Facio. Como se observa en el Cuadro 8, mientras que en general los patrones son similares a los descritos para toda la población de estudiantes, algunas particularidades pueden encontrarse en ciertas 
Aplicación del análisis de sobrevivencia al estudio del tiempo requerido para graduarse en educación superior: el caso costarricense

facultades. Así encontramos que en la Facultad de Letras el riesgo de graduarse por poseer beca es de 2,86 veces con respecto a los que no tienen, efecto muy superior al encontrado en el resto de la universidad; mientras que en otras escuelas la variable no resulta significativa. En cuanto a la nota de admisión, la Facultad de Ciencias Básicas y la Facultad de Ingeniería presentan aumentos en el riesgo de graduarse por cada 100 puntos superiores al 100\%, mientras en otras como en Medicina, Educación y Derecho, el efecto no es significativo.

Cuadro 8. Coeficientes de la regresión de Cox por facultad. Universidad de Costa Rica

\begin{tabular}{|c|c|c|c|c|c|c|}
\hline \multirow{2}{*}{ Variables } & \multirow{2}{*}{ B } & \multirow{2}{*}{$\exp (B)$} & \multirow{2}{*}{$\operatorname{se}(B)$} & \multirow{2}{*}{ Sig } & \multicolumn{2}{|c|}{ Intervalos de Confianza } \\
\hline & & & & & Inferior & Superior \\
\hline \multicolumn{7}{|l|}{ Artes } \\
\hline Colegio & $-0,029$ & 0,971 & 0,187 & & 0,674 & 1,400 \\
\hline Beca & 0,288 & 1,334 & 0,186 & & 0,928 & 1,920 \\
\hline Admisión & 0,404 & 1,497 & 0,119 & 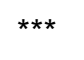 & 1,185 & 1,891 \\
\hline Sexo & $-0,339$ & 0,713 & 0,158 & * & 0,523 & 0,971 \\
\hline \multicolumn{7}{|l|}{ Letras } \\
\hline Colegio & $-0,163$ & 0,850 & 0,160 & & 0,621 & 1,163 \\
\hline Beca & 1,052 & 2,862 & 0,164 & $\star * *$ & 2,077 & 3,945 \\
\hline Admisión & 0,294 & 1,342 & 0,114 & $* *$ & 1,074 & 1,677 \\
\hline Sexo & $-0,684$ & 0,505 & 0,146 & $* \star \star$ & 0,379 & 0,672 \\
\hline \multicolumn{7}{|c|}{ Ciencias Básicas } \\
\hline Colegio & $-0,028$ & 0,972 & 0,164 & & 0,705 & 1,341 \\
\hline Beca & 0,162 & 1,176 & 0,163 & & 0,855 & 1,618 \\
\hline Admisión & 1,043 & 2,838 & 0,104 & $* * *$ & 2,313 & 3,482 \\
\hline Sexo & $-0,562$ & 0,570 & 0,135 & $* * *$ & 0,437 & 0,743 \\
\hline \multicolumn{7}{|l|}{ Derecho } \\
\hline Colegio & $-0,062$ & 0,940 & 0,147 & & 0,704 & 1,255 \\
\hline Beca & 0,238 & 1,269 & 0,145 & & 0,955 & 1,686 \\
\hline Admisión & 0,129 & 1,138 & 0,095 & & 0,945 & 1,369 \\
\hline Sexo & $-0,360$ & 0,697 & 0,121 & $* *$ & 0,551 & 0,883 \\
\hline \multicolumn{7}{|l|}{ Educación } \\
\hline Colegio & $-0,062$ & 0,940 & 0,147 & & 0,704 & 1,255 \\
\hline Beca & 0,238 & 1,269 & 0,145 & & 0,955 & 1,686 \\
\hline Admisión & 0,129 & 1,138 & 0,095 & & 0,945 & 1,369 \\
\hline Sexo & $-0,360$ & 0,697 & 0,121 & $* *$ & 0,551 & 0,883 \\
\hline
\end{tabular}


Aplicación del análisis de sobrevivencia al estudio del tiempo requerido para graduarse en educación superior: el caso costarricense

\begin{tabular}{|c|c|c|c|c|c|c|}
\hline \multirow{2}{*}{ Variables } & \multirow{2}{*}{ B } & \multirow{2}{*}{$\exp (B)$} & \multirow{2}{*}{$\mathrm{se}(\mathrm{B})$} & \multicolumn{3}{|c|}{ Intervalos de Confianza } \\
\hline & & & & Sig & Inferior & Superior \\
\hline \multicolumn{7}{|c|}{ Ciencias Económicas } \\
\hline Colegio & $-0,231$ & 0,794 & 0,069 & 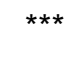 & 0,694 & 0,909 \\
\hline Beca & 0,494 & 1,639 & 0,068 & $\star * \star$ & 1,435 & 1,873 \\
\hline Admisión & 0,256 & 1,291 & 0,045 & $* * *$ & 1,183 & 1,409 \\
\hline Sexo & $-0,478$ & 0,620 & 0,055 & $* * *$ & 0,557 & 0,690 \\
\hline \multicolumn{7}{|c|}{ Ciencias Sociales } \\
\hline Colegio & 0,036 & 1,036 & 0,088 & & 0,872 & 1,231 \\
\hline Beca & 0,489 & 1,631 & 0,087 & $* * *$ & 1,375 & 1,935 \\
\hline Admisión & 0,546 & 1,726 & 0,050 & $* * *$ & 1,563 & 1,905 \\
\hline Sexo & $-0,663$ & 0,515 & 0,079 & $* * *$ & 0,441 & 0,601 \\
\hline \multicolumn{7}{|l|}{ Ingeniería } \\
\hline Colegio & $-0,110$ & 0,896 & 0,111 & & 0,721 & 1,113 \\
\hline Beca & 0,344 & 1,411 & 0,109 & $* *$ & 1,141 & 1,746 \\
\hline Admisión & 0,795 & 2,215 & 0,067 & $* * *$ & 1,943 & 2,525 \\
\hline Sexo & $-0,228$ & 0,796 & 0,104 & * & 0,649 & 0,976 \\
\hline \multicolumn{7}{|l|}{ Medicina } \\
\hline Colegio & $-0,017$ & 0,983 & 0,120 & & 0,778 & 1,243 \\
\hline Beca & 0,134 & 1,143 & 0,120 & & 0,903 & 1,446 \\
\hline Admisión & $-0,052$ & 0,949 & 0,064 & & 0,836 & 1,077 \\
\hline Sexo & $-0,508$ & 0,602 & 0,113 & $* * *$ & 0,482 & 0,751 \\
\hline \multicolumn{7}{|c|}{ Agroalimentarias } \\
\hline Colegio & $-0,017$ & 0,983 & 0,199 & & 0,666 & 1,450 \\
\hline Beca & 0,492 & 1,636 & 0,195 & * & 1,116 & 2,398 \\
\hline Admisión & 0,306 & 1,358 & 0,148 & * & 1,016 & 1,815 \\
\hline Sexo & 0,025 & 1,026 & 0,156 & & 0,755 & 1,394 \\
\hline
\end{tabular}

\section{DISCUSIÓN}

Tal y como se ilustra en el presente estudio, la aplicación de las herramientas de análisis de sobrevivencia utilizando el estimador KM y la regresión de Cox, se constituye en una alternativa estadística útil y adecuada para el estudio de los tiempos de graduación. La información que brinda este tipo de análisis es claramente superior a 
Aplicación del análisis de sobrevivencia al estudio del tiempo requerido para graduarse en educación superior: el caso costarricense

la que podría obtenerse mediante el cálculo de las medias. En el caso particular de la Universidad de Costa Rica, las curvas de sobrevivencia de KM mostraron estimaciones del tiempo de graduación relativamente altas en comparación con lo proyectado de acuerdo a la planificación curricular de las carreras. Así, al cabo de ocho años (16 semestres), el modelo estima que solo el $37 \%$ de los estudiantes habrían concluido sus estudios de bachillerato, en contraste con los planes de estudio que organizan sus carreras en solo ocho ciclos lectivos. De este modo la duración estimada es del doble del tiempo estipulado por los planes de estudio. Sin embargo esta estimación no contempla el hecho de que algunos de estos estudiantes en realidad no continuaron sus estudios.

A partir del mismo modelo se estimó que el porcentaje de estudiantes en dicha condición de deserción sería de aproximadamente 38\%. Al eliminar ese grupo de estudiantes de los cálculos, la estimación ajustada arroja un $65 \%$ que completarían sus estudios al cabo de ocho años. Este nuevo cálculo continúa mostrando un tiempo relativamente alto para completar los estudios de bachillerato, por ejemplo si lo comparamos con estadísticas de otros países. Así, Astin y Oseguera (2005) reportan que en las instituciones de los Estados Unidos al cabo de seis años, se obtiene una graduación del $50 \%$, mientras que según nuestras estimaciones en la UCR este valor se alcanzaría después de ocho años.

Respecto de los factores asociados con el tiempo de graduación, se encontró que las mujeres muestran tiempos más cortos que los hombres, y que las sedes regionales tienen tiempos inferiores que la sede central. Mediante la aplicación de la regresión de Cox se encontró que la nota de admisión afectaba el tiempo de graduación, de manera que los (las) estudiantes con mejores promedios tienden a finalizar antes sus carreras. Igualmente se encontró un efecto significativo del colegio de procedencia, sin embargo la dirección de esta relación fue contraria a la esperada, ya que aquellos estudiantes provenientes de colegios de pago mostraron tendencia a tardar más en finalizar sus carreras. Este patrón parece explicarse por el hecho de que la Universidad de Costa Rica provee de beca a los estudiantes de condición socioeconómica baja. Adicionalmente, existe cierta presión para los estudiantes que gozan de esta ayuda, ya que de no mostrar avance en sus programas, la Universidad 
podría retirarles dicha beca. Una vez incluida la variable correspondiente a la presencia de beca de asistencia socioeconómica, el efecto del colegio se muestra significativo en la dirección esperada. Al estratificar por sede, esta situación se mantiene únicamente en la sede central, ya que el efecto del colegio no aparece como significativo en las demás, lo cual tiene sentido por la menor presencia de colegios privados (de pago) en la periferia del país. Es importante señalar que la procedencia de colegio privado ha mostrado ser un excelente predictor de nivel socioeconómico alto.

En general, estos hallazgos sugieren que la prueba de admisión es un buen predictor de la duración de la carrera, lo cual puede ser interpretado como evidencia de validez psicométrica de la misma. Es cierto que el efecto de la PAA en tanto indicador de aptitud puede estar confundido con el nivel socioeconómico del(a) estudiante (Montero y Villalobos, 2004), sin embargo el efecto se mantiene al incluir el colegio de proveniencia y es conocido el hecho de que la proveniencia de colegios privados es un buen predictor de nivel socioeconómico (Fernández y Del Valle, 2013). De todos modos hay que considerar el hecho de que la nota de admisión se utiliza también como criterio para que los estudiantes escojan la carrera de sus preferencias, de modo que estudiantes con bajas puntuaciones pueden terminar empadronándose en carreras que no sean las de su interés, de modo que la falta de motivación pueda ser un factor que incida en mayores tiempos para concluir los estudios.

Como se dijo anteriormente, el tipo de colegio de proveniencia resulta un buen indicador del nivel socioeconómico. De hecho, esta variable es utilizada como uno de los indicadores que mejor funcionan para predecirlo, con fines de asignación de becas en la UCR. Al respecto esta investigación provee evidencia de que el sistema de beca parece tener un efecto atenuador con respecto de la condición socioeconómica de los estudiantes, ya que al considerarse ambas variables simultáneamente en regresiones de Cox se muestran efectos en direcciones opuestas. Ya que la asignación de beca está relacionada de forma inversa con el nivel socioeconómico y, específicamente con el tipo de colegio de procedencia, se puede explicar el que al incluirse únicamente esta última variable no se obtenga un efecto estadísticamente significativo.

\section{REFERENCIAS}


Astin, A.W., \& Oseguera, L. (2005). Degree Attainment Rates at American Colleges and Universities. Los Angeles, CA: Higher Education Research Institute at UCLA.

Astin, A.W., \& Oseguera, L. (2012). Pre-College and Institutional Influences on Degree Attainment. Seidman, A. (Ed.), College Student Retention: Formula for Student Success (245-277). Washington, DC: American Council on Education/Praeger Series on Higher Education.

Bergeron, D. y Flores, A. (2015). Improving College Access and Degree Attainment Through an Early Guarantee of Federal Financial Aid. Washington, D.C: Center for American Progress.

Carrell, S. E., \& Sacerdote, B. (2013). Why do college-going interventions work? Cambridge, MA: National Bureau of Economic Research.

Carruthers, C.K. \& Özek, U. (2016). Losing HOPE: Financial aid and the line between college and work. Economics of education review, 53, 1-15.

CONARE (1993). Perfil de los graduados de Educación Superior Universitaria Estatal. San José, Costa Rica: OPES.

CONARE (2001). La situación laboral y otras características de los graduados de 1998 de las Universidades Estatales. San José, Costa Rica: OPES.

Cox, D.R. (1972). Regression Models and Life-Tables. Journal of the Royal Statistical Society, 34, 187-220.

Cubero, V. M. \& Longhi, E. (1995). Validez Predictiva de los Puntajes de Admisión y Confiabilidad de la Prueba de Aptitud Académica. Actualidades en Psicología, 11, 88-89.

Engle, J. y O'Brien, C. (2007). Demography is not destiny: Increasing the graduation rates of low-income college students' at large public universities. Washington: The Pell Institute.

Fernández, A. y Del Valle, R. (2013). Desigualdad educativa en Costa Rica: La brecha entre estudiantes de colegios públicos y privados. Análisis con los resultados de la evaluación internacional PISA. Revista CEPAL, 111, 37-57.

Gómez, G (2004) Análisis de supervivencia. Barcelona, España. 
Hosmer, D.W., Lemeshow, S. y Sturdivant, R.X. (2013). Applied logistic regression. Hoboken, NJ: John Wiley.

Jiménez-Alfaro, K. y Morales-Fernández, E (2010). Validez predictiva del Promedio de Admisión de la Universidad de Costa Rica y sus componentes. Actualidades en Psicología, 23, 21-55.

Kaplan, E. L. \& Meier, P (1958). Nonparametric Estimation from Incomplete Observations. Journal of the American Statistical Association, 53, 457-481.

Montero, E y Villalobos, J. (2004). Estudio Comparativo del Promedio de Admisión a la Universidad de Costa Rica y sus Componentes: Aplicación del Año 2001. San José, Costa Rica: Programa Permanente de la Prueba de Aptitud Académica, Instituto de Investigaciones Psicológicas, Universidad de Costa Rica.

Montero, E., Rojas-Rojas, E., Negrín-Hernández, M., y Francis-Salazar, S. (2015). Efecto de una capacitación sobre los puntajes de la prueba de admisión de la Universidad de Costa Rica: una aproximación bayesiana. Actualidades en Psicología, 119, 115-139.

Programa Estado de la Nación (2015). Vigésimo primer Informe Estado de la Nación en Desarrollo Humano Sostenible. San José, Costa Rica: PEN.

Reglamento del Proceso de Admisión mediante la Prueba de Aptitud Académica (2003). Gaceta Universitaria Universidad de Costa Rica, San José, Costa Rica, 7 de marzo del 2003. 